\title{
Characterizing and mitigating vibrations for SCExAO
}

\author{
Julien Lozi ${ }^{\mathrm{a}}$, Olivier Guyon ${ }^{\mathrm{a}, \mathrm{b}, \mathrm{c}, \mathrm{d}}$, Nemanja Jovanovic ${ }^{\mathrm{a}, \mathrm{e}}$, Garima Singh ${ }^{\mathrm{f}}$, Sean Goebel ${ }^{\mathrm{a}, \mathrm{g}}$, \\ Barnaby Norris ${ }^{\mathrm{h}}$, and Hirofumi Okita ${ }^{\mathrm{a}}$ \\ ${ }^{a}$ National Astronomical Observatory of Japan, Subaru Telescope, 650 North A'ohōkū Place, \\ Hilo, HI 96720, U.S.A. \\ ${ }^{\mathrm{b}}$ Steward Observatory, University of Arizona, Tucson, AZ 85721, U.S.A. \\ ${ }^{\mathrm{c}}$ College of Optical Sciences, University of Arizona, Tucson, AZ 85721, U.S.A. \\ ${ }^{\mathrm{d} A s t r o b i o l o g y}$ Center of NINS, 2-21-1, Osawa, Mitaka, Tokyo, 181-8588, Japan \\ ${ }^{\mathrm{e}}$ Department of Physics and Astronomy, Macquarie University, NSW 2109, Australia \\ ${ }^{\mathrm{f} J}$ Jet Propulsion Laboratory, 4800 Oak Grove Drive, MS 183-901, Pasadena, CA 91109, U.S.A. \\ gInstitute for Astronomy, University of Hawai'i, 640 North A'ohōkū Place, Hilo, HI 96720, \\ U.S.A. \\ ${ }^{\mathrm{h}}$ Sydney Institute for Astronomy, Institute for Photonics and Optical Science, School of \\ Physics, University of Sydney, NSW 2006, Australia
}

\begin{abstract}
The Subaru Coronagraphic Extreme Adaptive Optics (SCExAO) instrument, under development for the Subaru Telescope, has currently the fastest on-sky wavefront control loop, with a pyramid wavefront sensor running at $3.5 \mathrm{kHz}$. But even at that speed, we are still limited by low-frequency vibrations. The current main limitation was found to be vibrations attributed mainly to the rotation of the telescope. Using the fast wavefront sensors, cameras and accelerometers, we managed to identify the origin of most of the vibrations degrading our performance. Low-frequency vibrations are coming from the telescope drive in azimuth and elevation, as well as the elevation encoders when the target is at transit. Other vibrations were found at higher frequency coming from the image rotator inside Subaru's adaptive optics facility AO188.

Different approaches are being implemented to take care of these issues. The PID control of the image rotator has been tuned to reduce their high-frequency contribution. We are working with the telescope team to tune the motor drives and reduce the impact of the elevation encoder. A Linear Quadratic Gaussian controller (LQG, or Kalman filter) is also being implemented inside SCExAO to control these vibrations. These solutions will not only improve significantly SCExAOs performance, but will also help all the other instruments on the Subaru Telescope, especially the ones behind AO188. Ultimately, this study will also help the development of the TMT, as these two telescopes share very similar drives.
\end{abstract}

Keywords: Extreme Adaptive Optics, Vibrations, Control, LQG, Accelerometers

\section{INTRODUCTION}

Vibrations are one of the major source of degradation in current high contrast imaging systems. ${ }^{1-4}$ The requirement in pre-coronagraphic tip/tilt correction is usually about a few milliarcseconds for instruments on 8-m class telescopes. It will be even less with the next generation of extremely large telescopes. This problem is also faced by the Subaru Coronagraphic Extreme Adaptive Optics (SCExAO) instrument, ${ }^{5,6}$ at the Subaru Telescope. Vibrations can be introduced by any element in the optical train, from the primary mirror of the telescope to the last optics focusing on the coronagraphic mask. Their origin can come from the telescope itself, its resonance frequencies, its drive system, or its pointing loop control. Other elements can also introduce vibrations, like any cryogenic pump, vacuum pump or other motors, close to the optical path. Subaru Telescope vibrations where

Further author information: (Send correspondence to J.L.)

J.L.: E-mail: lozi@naoj.org, Telephone: 18089345949

Adaptive Optics Systems V, edited by Enrico Marchetti, Laird M. Close, Jean-Pierre Véran, Proc. of SPIE Vol. 9909, 99090J (c) 2016 SPIE - CCC code: 0277-786X/16/\$18 - doi: 10.1117/12.2233040 
already studied and reduced in the past, ${ }^{7}$ using accelerometers and by tuning the gains in the pointing control loop. With the high sensitivity of SCExAO, this tuning is not enough to cancel most of the telescope vibrations. In Sec. 2, we present how vibrations affect SCExAO's science data. Then Sec. 3 present our analysis of the different sources of vibrations degrading our results. Finally Sec. 4 presents experimental on-sky results of a Linear Quadratic Gaussian (LQG) controller implemented on SCExAO to perform a real-time correction of the vibrations.

\section{IMPACT OF VIBRATIONS}

\subsection{Degradation in Point Spread Function Quality}

The main metric of the correction quality of the Extreme Adaptive Optics (ExAO) of SCExAO is the Point Spread Function (PSF) quality, quantified either in Strehl ratio (SR) or residual wavefront error. During SCExAO's observations, a few fast Near-Infrared (NIR) cameras can be used to monitor rapid changes in the PSF quality. The main one is our IR internal camera. This camera, equipped with a $320 \times 256$ InGaAs detector, can acquire images with a maximum cadence of $170 \mathrm{~Hz}$.

Figure 1 (left) presents the instantaneous SR measured with our NIR internal camera at $170 \mathrm{~Hz}$, over 1000 frames - or about $6 \mathrm{~s}-$-, when only AO188's control loop is closed. Most of the variations seen here are due to the residual turbulence left uncorrected by AO188. The average SR calculated with this data set is $47.3 \%$.
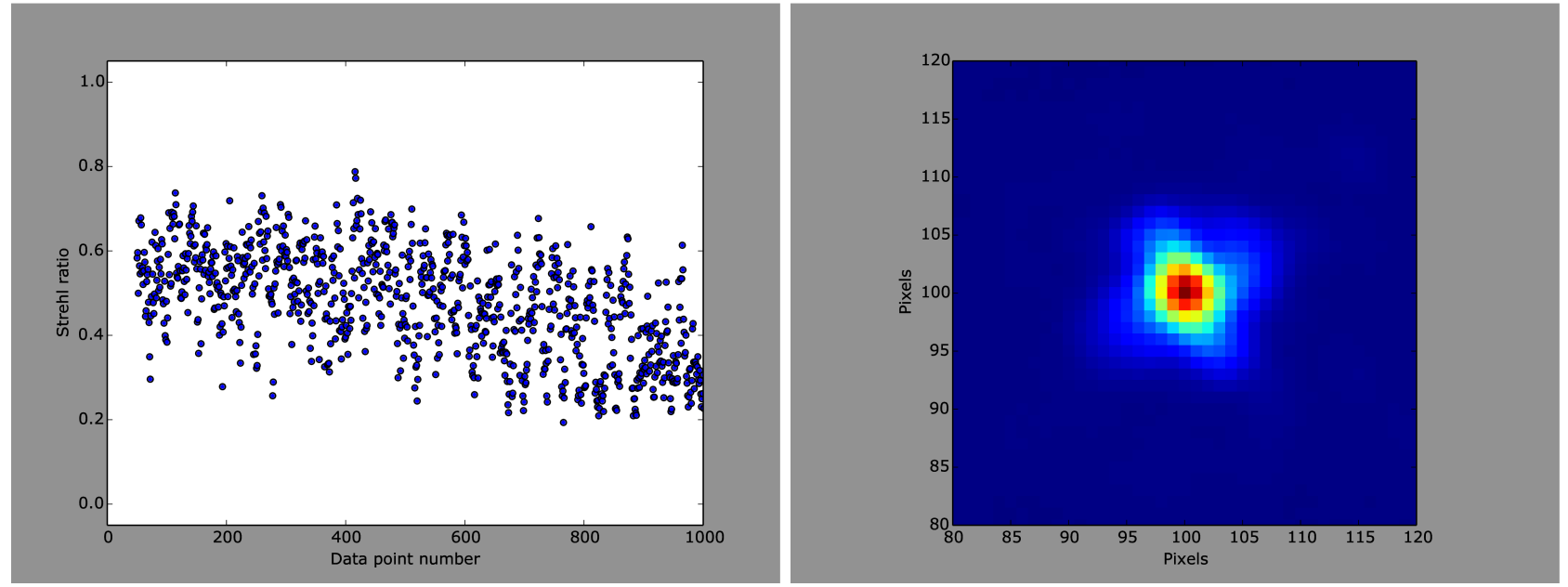

Figure 1. Evolution of the instantaneous SR with AO188 only (left), and averaged PSF over 1000 frames (right).
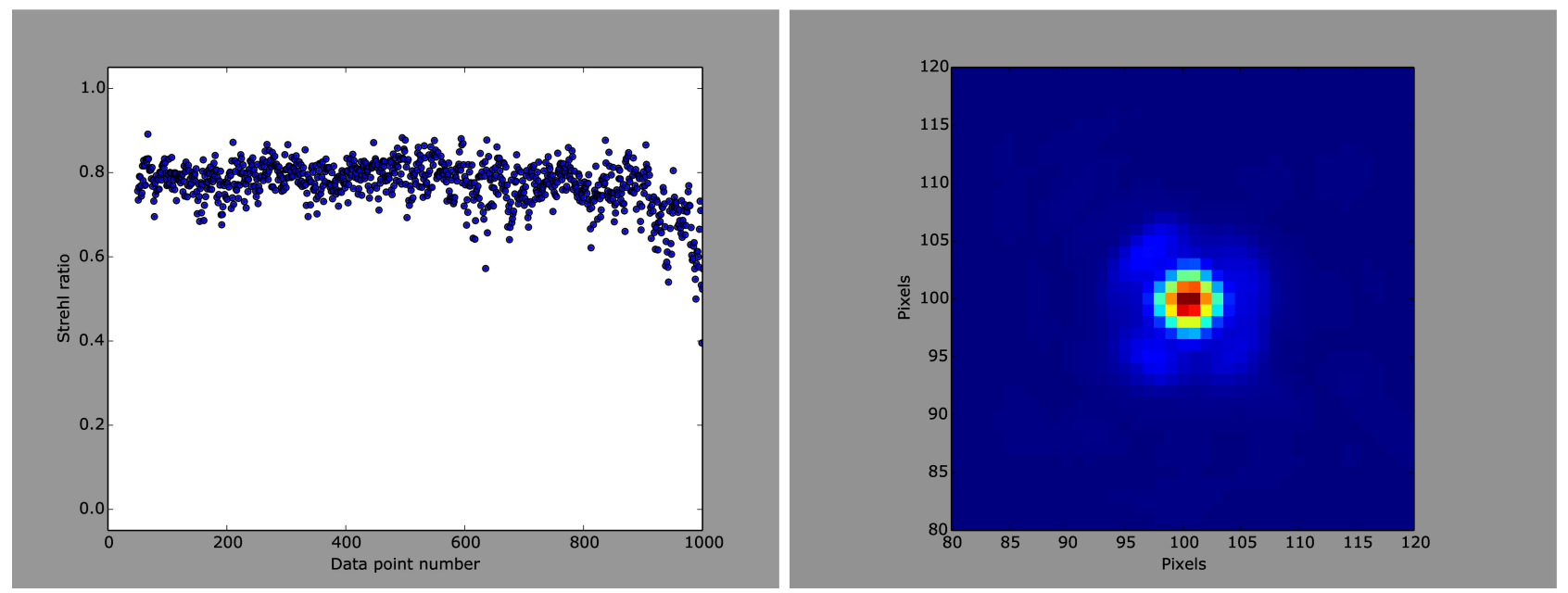

Figure 2. Evolution of the instantaneous SR with the PyWFS (left), and averaged PSF over 1000 frames (right). 
Using the averaged PSF over the 1000 images presented in Fig. 1 (right), we performed the same SR calculation than for each individual image, and found a value of $28.8 \%$. This result indicates that the SR is reduced by $18.5 \%$ just from low frequency $(\ll 170 \mathrm{~Hz})$ variations, i.e. vibrations.

We performed the same analysis when the ExAO of SCExAO was in closed-loop. Figure 2 presents the same analysis than in Fig. 1. When the loop was closed for this data set, the instantaneous SR had smaller variations, around a mean value of $78.6 \%$. We are still not quite reaching SCExAO's goal of $90+\%$. But when the frames are averaged to simulate a long exposure time, the measured SR is now $60.9 \%$. So once again, $17.7 \%$ of the SR is lost due to vibrations.

These vibrations, degrading the PSF quality, will also increase the stellar leakages around the coronagraphic mask, therefore degrading the final contrast in the science images.

\subsection{Contrast reduction in Interferometric Instruments}

SCExAO has the unique property to host a few interferometric and fiber injection modules in visible wavelengths: ${ }^{6}$

- VAMPIRES,$^{8}$ a high precision polarimetric instrument using non-redundant masks;

- FIRST, ${ }^{9}$ an interferometer and spectrometer, using non-redundant remapping of the pupil with single mode fibers;

- RHEA, a high-resolution 9-element integral field spectrograph, using single mode injection in the focal plane;

- NULLER, a visible nulling interferometer, also using single mode fiber injection.

Since they are working in visible wavelengths, these modules are also very sensitive to vibrations in the optical path. Figure 3 presents VAMPIRES polarimetric data in the Fourier plane for a typical target, when vibrations are small (left), and for Polaris, a target where strong vibrations are occurring (right). In this example of a strong vibration, we can notice that some spatial frequencies are washed out in the Fourier plane. It is therefore much harder to reconstruct the object observed. Similar problems occur in the presence of vibrations for the other visible modules. Fringe contrasts get reduced for interferometers, while coupling degrades in single mode fibers.

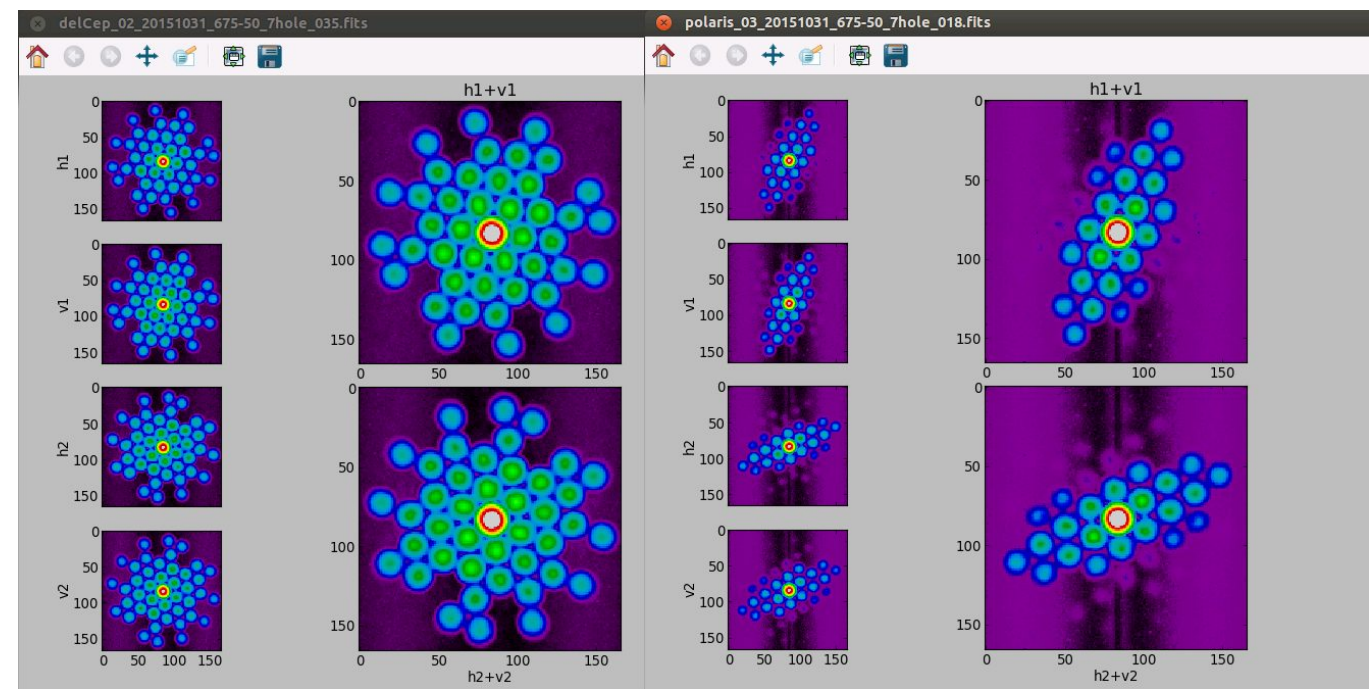

Figure 3. Fourier plane of the polarization components in VAMPIRES, when vibrations are low (left) and when vibrations are very high (right). 


\section{MEASURING VIBRATIONS OF THE SUBARU TELESCOPE}

\subsection{Metrology of SCExAO to measure vibrations}

SCExAO has several high frame rate cameras that can be used to monitor the PSF motion inside the instrument. The PyWFS, measures about 1200 modes up to $3.5 \mathrm{kHz}$, including low-order modes like tip/tilt and focus. In addition, SAPHIRA ${ }^{10}(800 \mathrm{~Hz})$, as well as the internal NIR camera $(170 \mathrm{~Hz})$, can be used to track the pointing accuracy. Finally, the LLOWFS, ${ }^{11,12}$ also running at $170 \mathrm{~Hz}$, can monitor low-order aberrations inside the coronagraph left uncorrected by the PyWFS. The image rotator inside AO188 is placed in a fixed pupil mode, so elevation and azimuth are constant axes on the various detectors, at $37^{\circ}$ of the cardinal axes of the instrument.

Using the fast NIR camera SAPHIRA, which is built with a HgCdTe avalanche photodiode array and runs at about $800 \mathrm{~Hz}$ on a subframed window, we can track precisely the motion of the PSF, and analyze the vibration frequencies using the Power Spectral Density (PSD) of the position. Figure 4 presents such a PSD of the PSF position, with the axes rotated to match the elevation and azimuth of the telescope. In this figure, we notice a lot of vibrating frequencies, from about $5 \mathrm{~Hz}$ to $300 \mathrm{~Hz}$, with various amplitudes. Since vibration frequencies usually depend on the weight of the moving optics and mounts, we expect the lowest frequencies $(\lesssim 10 \mathrm{~Hz})$ to come from the telescope, while higher frequencies come from smaller optics and mounts.

During on-sky observations, we used the coronagraph and the LLOWFS, and recorded vibrations around the transit (maximum elevation) of the star in the sky. The result is presented in Fig. 5, in azimuth (left) and elevation (right). The observed target did not have enough flux to for the LLOWFS to work at full speed. In this case, it was running at $20 \mathrm{~Hz}$.

Over the few hours of a typical observation, the vibrating frequencies tend to evolve. So a classical PSD of the tip/tilt measurements would either only show an instantaneous view of the conditions if a small sample is used, or smear out any shifting frequency and keep only fixed vibrations. This is why in Fig. 5 and the next ones, we present an evolution of the PSD: each line of the 2D image is a PSD of a sliding sample of data. The samples contain 1000 points each, and are separated by 500 points. In this case, vibrations with constant frequencies are visible as straight vertical lines, while evolving vibrations are visible as curved lines.

In Fig. 5 (left), we can see a slow evolving vibration, with a maximum frequency at the time of transit $(t=1000 \mathrm{~s})$, and a harmonic at twice the frequency of the first one, but folded due to the Nyquist frequency of the LLOWFS $(10 \mathrm{~Hz})$. In Fig. 5 (right), more features are visible. After $t=2000 \mathrm{~s}$ is a slowly increasing vibration starting at $0 \mathrm{~Hz}$ at the transit, with a harmonic stronger than the first component at twice the frequency. But the strongest feature is happening around the transit time, between 4 and $6 \mathrm{~Hz}$. This feature is usually visible on most targets, and disrupts the quality of the scientific images. Since most post-processing techniques rely

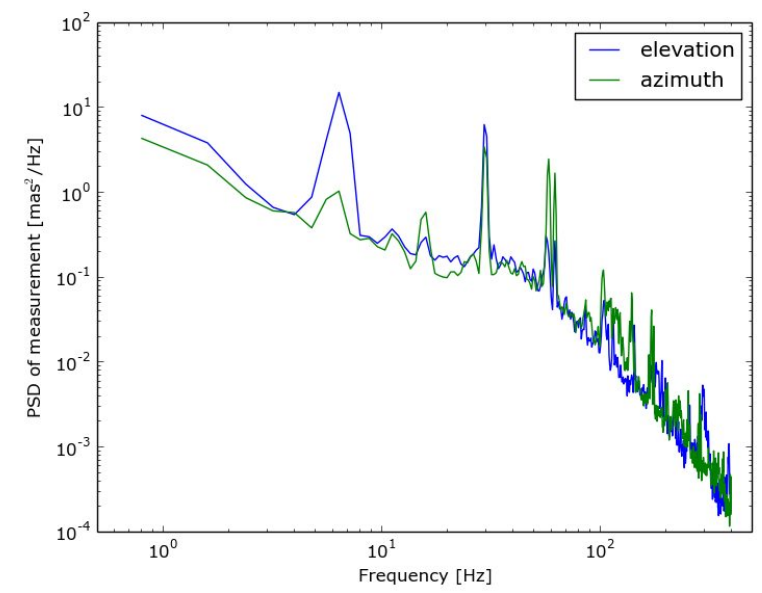

Figure 4. PSD of the PSF centroid measured in the fast NIR camera SAPHIRA. 

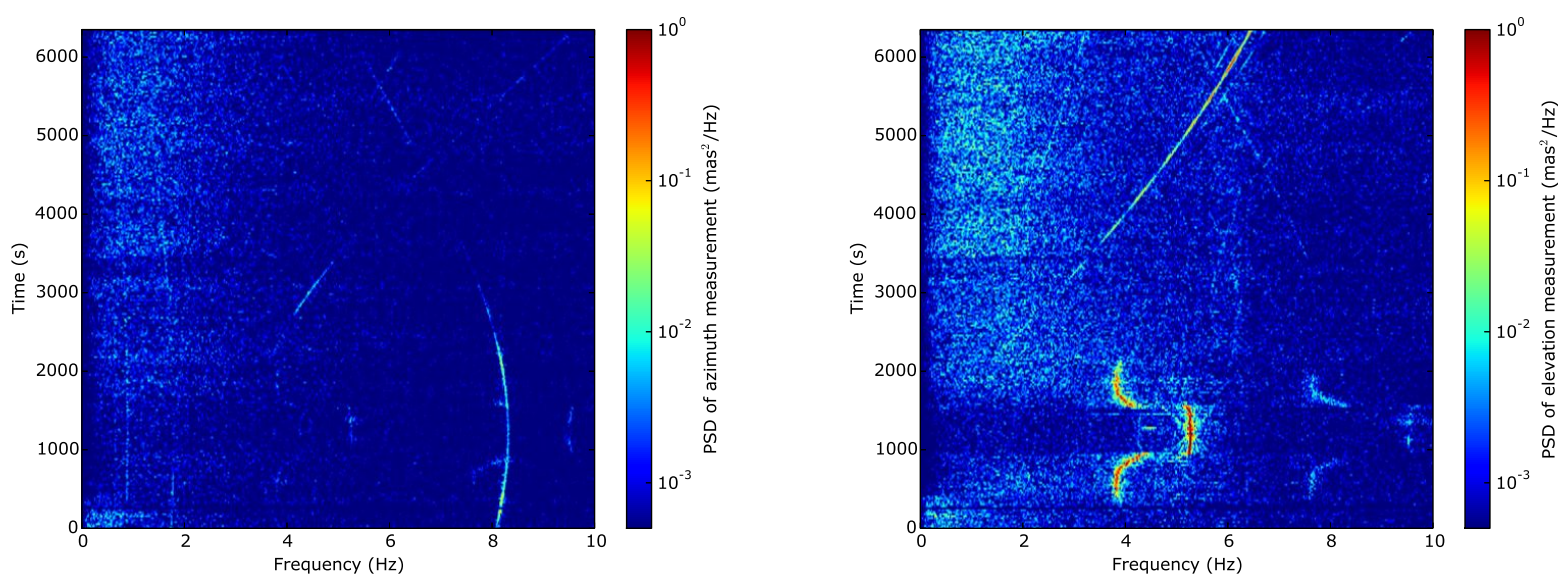

Figure 5. PSD evolution of LLOWFS data, in azimuth (left) and elevation (right). The transit occurs at $1200 \mathrm{~s}$.

on the field rotation of the planets or disks around the transit time, this vibration has a strong impact on the detection of exoplanets and other disk features around the targets.

All the vibrations observed here are clearly originating from the telescope, since they are at low frequencies and aligned with its rotation axes. When compared to the rotation speed of the telescope, it is clear the frequencies of the slow evolving features are proportional to that speed: the telescope's elevation rotates the fastest around the transit, while the azimuthal axis is almost static at that time.

\subsection{Monitoring the Telescope Vibrations Using Accelerometers}

To monitor telescope vibrations during observations, a set of three accelerometers were installed on one side of the top ring supporting the secondary mirror. These are the same accelerometers used for the study presented here. $^{7}$ The three accelerometers are placed orthogonally one from another, such as " $x$ " and " $y$ " are in the plane of the top ring, and " $z$ " is orthogonal to that plane. So the "z" accelerometer measures mostly the distance variation between the primary and secondary mirror. Due to the strong structure of the Subaru Telescope, that motion is actually negligible, so we will not show results from that accelerometer. The data is sampled at $60 \mathrm{~Hz}$, while the accelerometers have a maximum response of about $20 \mathrm{~Hz}$.
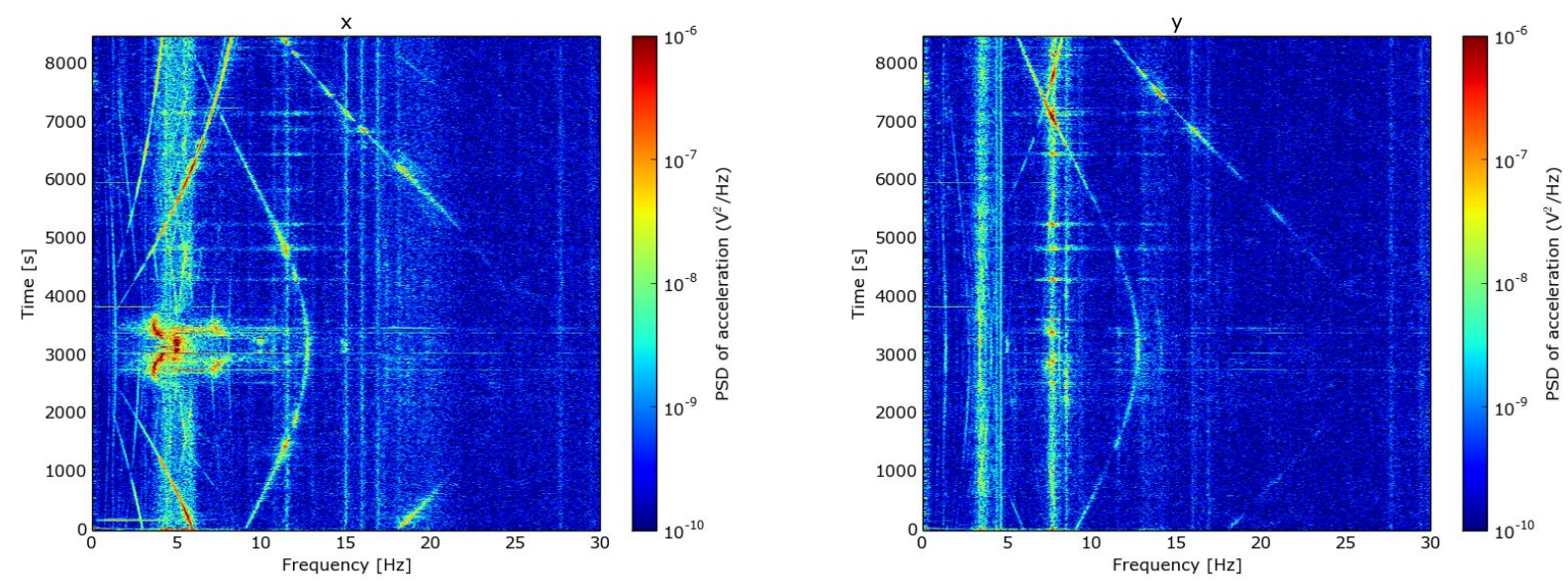

Figure 6. PSD evolution of the acceleration measured at the top ring of the telescope, for a $72^{\circ}$-elevation target, in $\mathrm{x}$ (left) and $\mathrm{y}$ (right). The transit is around $3200 \mathrm{~s}$. 

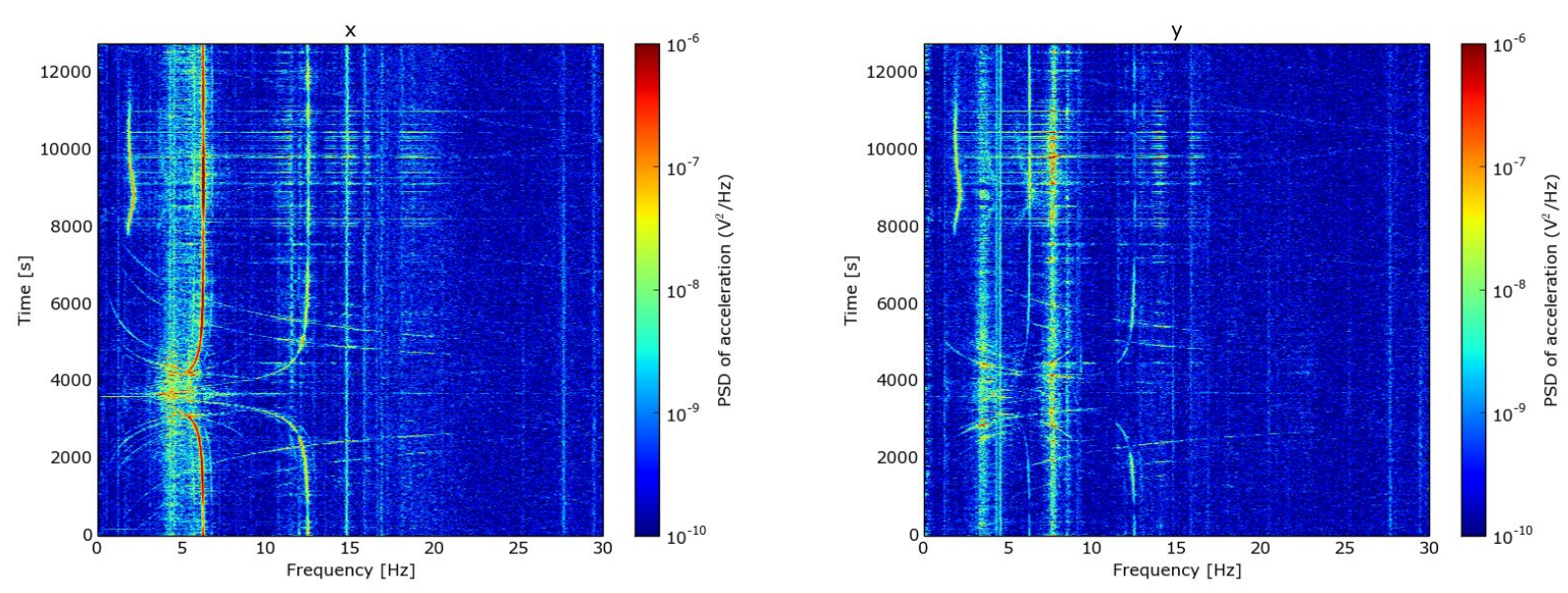

Figure 7. PSD evolution of the acceleration measured at the top ring of the telescope, for a $90^{\circ}$-elevation target, in $\mathrm{x}$ (left) and $\mathrm{y}$ (right). The transit is around $3800 \mathrm{~s}$.

Figures 6 and 7 present accelerometer measurements in $\mathrm{x}$ and $\mathrm{y}$, during the observations of two stars. The first target in Fig. 6 has a maximum elevation of $72^{\circ}$, while the second target in Fig. 7 has a maximum elevation of almost $90^{\circ}$.

Since the $\mathrm{x}$ and $\mathrm{y}$ axes do not correspond to the azimuth and elevation of the telescope, it is not trivial to decipher the origin of the vibrations observed here. But if we consider that these vibrations are proportional to the speed of the telescope rotations, then we can conclude that the vibrations with a maximum at the transit of the targets (respectively $t=3000 \mathrm{~s}$ and $t=4000 \mathrm{~s}$ ) are induced by the azimuthal rotation of the telescope. These vibrations are visible on both $\mathrm{x}$ and $\mathrm{y}$ axes. Similarly, the vibrations that have a 0 frequency at transit (zero speed) and an increasing frequency around it are induced by the elevational rotation of the telescope. These vibrations are mostly visible on the $\mathrm{x}$ axis. For a target transiting almost at zenith (Figure 6), the frequency variations are much faster around transit than for a lower target, which is expected.

Around the time of transit of both targets, we can see the same feature discovered in the LLOWFS data (Fig. 5). It has a duration that depends on the target transit elevation: it lasts a few dozens of minutes for a $72^{\circ}$-elevation star, and only a couple of minutes for a star transiting at zenith. It is now known that this features comes from a secondary control loop inside the elevation drive of the telescope. The encoders of Subaru have a pitch of $40 \mu \mathrm{m}$, corresponding to about 0.5 arcsec on-sky, which is bigger than the pointing precision of the telescope. So a secondary loop tracks the current sent to the motors, to achieve a higher pointing precision. When the star transits, the elevation of the telescope is quasi-static, putting more constraints on that secondary loop, creating this unique vibrating feature.

\subsection{Correlation Between Accelerometer Data and Pointing in SCExAO}

The metrology given by the PyWFS is extremely useful for vibration analysis, because it is the fastest $(3.5 \mathrm{kHz})$ and the most precise. Figure 8 presents a comparison between accelerometer data and PyWFS data in tip. The PyWFS data, taken in this experiment at $1.5 \mathrm{kHz}$, was binned to simulate the same $60-\mathrm{Hz}$ acquisition frequency than the accelerometers. This figure shows a very strong correlation between the two sets of data, meaning that the vibrations measured by the accelerometers are indeed also seen in the instrument. The vibration around the transit is visible on both data, as well as slow varying frequencies in both axes - the tip mode in the PyWFS being a combination of azimuth and elevation. The zone of higher noise around $5 \mathrm{~Hz}$ is also seen in both data. Vibrations going through this zone seem to be amplified, which means that it could correspond to an overshoot of the pointing control, or natural vibration modes of the telescope.

In a near future, the goal will be to use accelerometer data to perform an open-loop control by sending offsets to the tip/tilt mirror of AO188. The result presented here shows that there is indeed a strong correlation between measured vibrations and tip/tilt position inside the SCExAO instrument. 

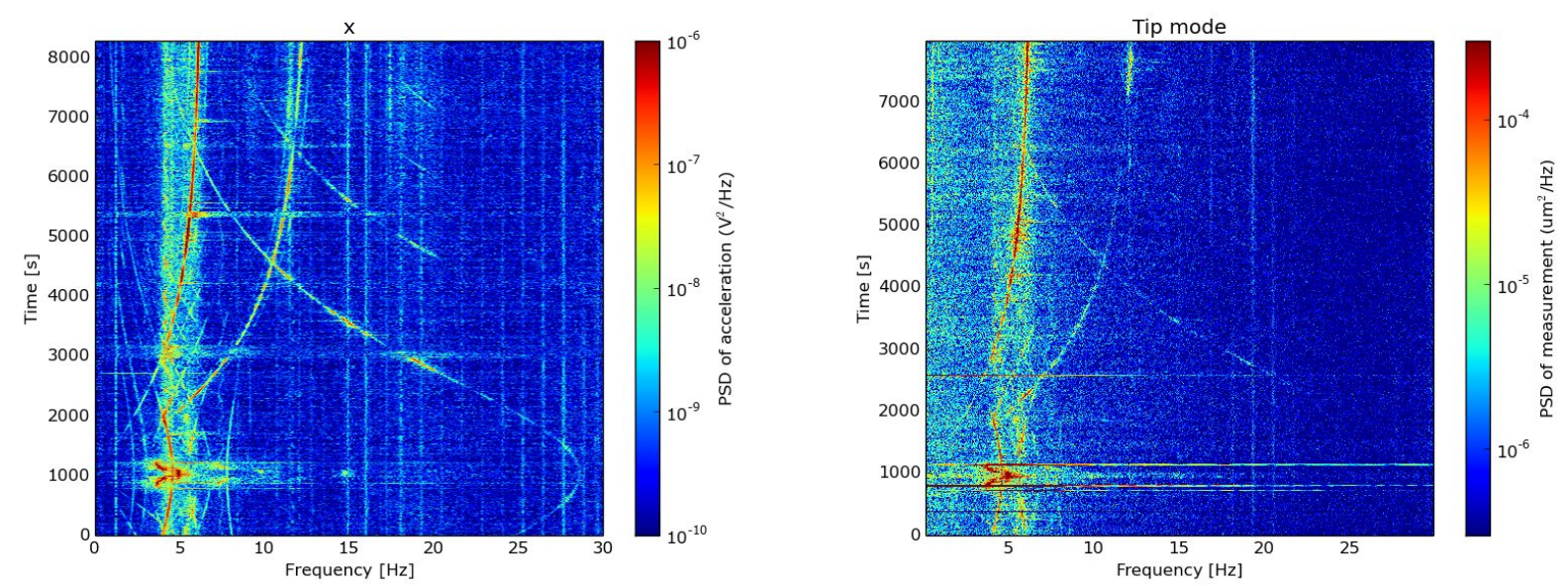

Figure 8. PSD evolution of accelerometer data (left) and PyWFS tip data (right). The transit is at $1000 \mathrm{s.}$

\subsection{Tuning the Image Rotator Controller}

A number of high frequency vibrations were seen in Fig. 4. We determined that most of them were actually coming from the rotation of the image rotator inside AO188. This device, composed of several mirrors, rotates during observations to keep a static pupil inside the instrument, while the field of view is rotating. Its rotation speed is linked with the rotation speed of the telescope, so the vibrations induced can be misinterpreted as telescope vibrations.

To reduce its impact, we decided to tune the control loop of the image rotator. The tuning was done by changing the control scheme from a discrete control to a continuous control of the rotation, and by reducing the gains of the PID controller. Figure 9 presents the result of this experiment. The tuning reduced significantly the amplitudes of the vibrations, especially after $100 \mathrm{~Hz}$. The PSD after tuning is only marginally higher than when the image rotator control is powered but static (red plot). The precision of the rotation was slightly degraded by reducing the gains, but still within the specifications.
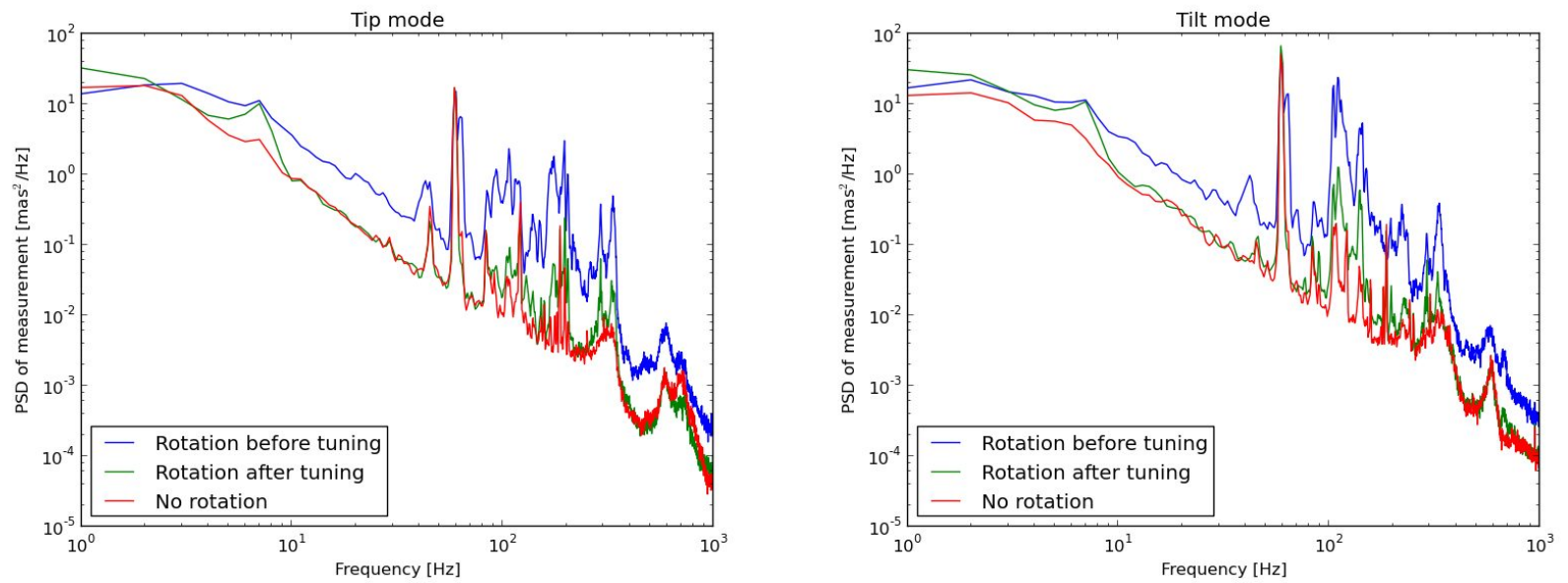

Figure 9. Impact on the PSD of tip (left) and tilt (right) of the tuning of the image rotator. 


\section{LINEAR QUADRATIC GAUSSIAN CONTROLLER}

\subsection{Architecture}

An analysis of the PyWFS modes shows that, unlike the Gemini Planet Imager, ${ }^{4}$ there is no significant vibrations in focus, and other centro-symmetric modes. Only tip and tilt — and at a lower level the astigmatism modesare disturbed by vibrations. Since they are the major contributors, for now we will just correct vibrations in tip/tilt.

The architecture of SCExAO's wavefront control combines multiple wavefront sensors sending commands to the same Deformable Mirror (DM) ${ }^{6,13}$ For the tip/tilt modes, a first stage correction is performed by the tip/tilt loop of AO188, with AO188's tip/tilt mount. A second stage of correction is then performed using the PyWFS and SCExAO's DM. Finally, if a coronagraph is used, The LLOWFS will correct non-common path tip/tilt residuals behind the coronagraphic mask. Since the vibrations are mostly introduced before the light enters SCExAO, we will focus on correcting them with the PyWFS.

To mitigate vibrations, we decided to implement a Linear Quadratic Gaussian (LQG) controller, ${ }^{14,15}$ with a real-time model identification of the vibrations. ${ }^{16}$ The final architecture is similar to the tip/tilt mitigation scheme of SPHERE. ${ }^{17}$ A Kalman-based predictive control, the LQG controller, calculates the optimal command to send to the DM, using a model of the disturbance. This model is updated in real-time to take into account evolving frequencies of the vibrations. The loop control is described in Sec. 4.3, while the model identification is described in Sec. 4.2 .

\subsection{Identification of the Model}

Using the residual tip/tilt measurements and the commands sent to the DM, it is possible to estimate in real-time the open-loop tip/tilt values. These pseudo-open-loop values generated during the loop control are used to built the model of the disturbance. This model, as described in, ${ }^{16}$ is composed of multiple second-order auto-regressive (AR2) models: one for the atmospheric turbulence —or in our case, a residual turbulence left by AO188's tip/tilt control- , and one for each vibration. The damping coefficient for the turbulence is then pretty large $(>1)$, while the ones for each vibrations are very small $(\ll 1)$, simulating sharp peaks. The model has to be updated often to take care of the changing frequencies of the vibrations.
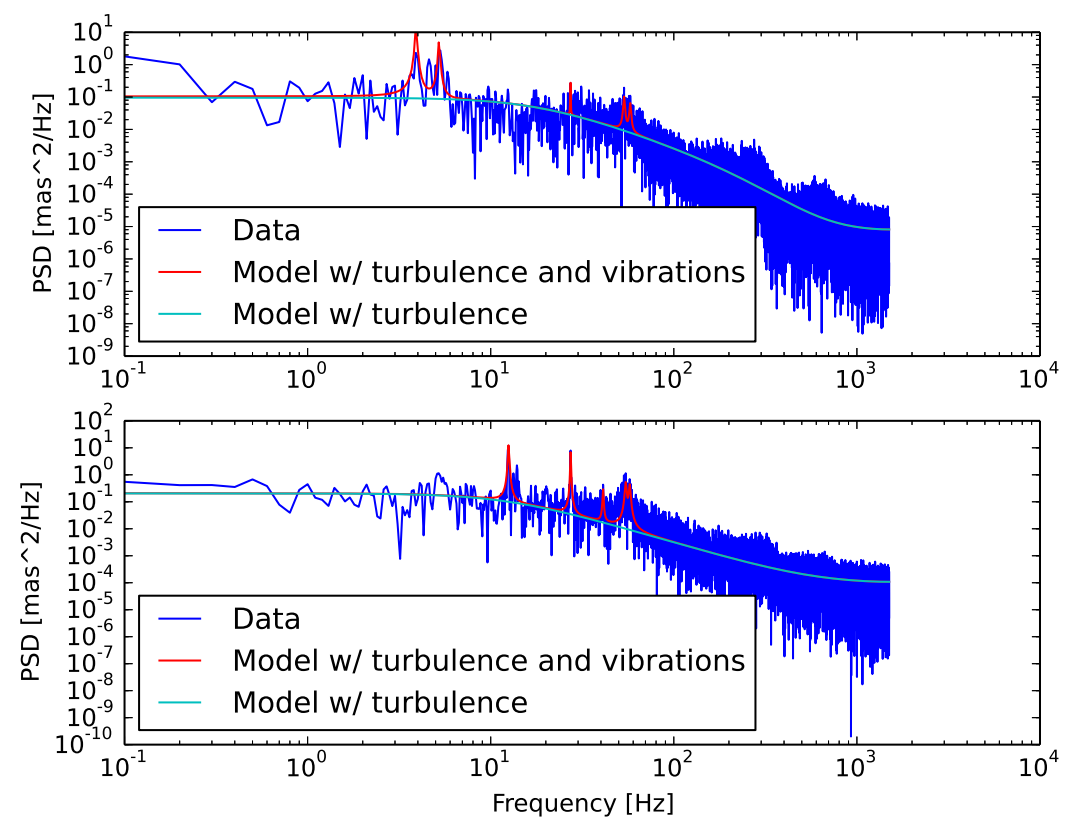

Figure 10. Identification of the model for turbulence and 5 vibrations on pseudo open-loop data, in tip (top) and tilt (bottom). 
Figure 10 presents the result of the identification procedure. The blue data corresponds to the PSD of pseudo-open-loop data in tip and tilt. The cyan plot then corresponds to the AR2 model of the turbulence, fitted to the PSD after removing the vibrations. Finally, the red plot corresponds to the final model, containing in this case the model of the turbulence and 5 AR2 models fitting the 5 strongest vibrations.

The pseudo-open-loop data is calculated in real-time and stored in a buffer of a few 10,000 iterations. This buffer is read every minute or so, and a model is identified using this data. The calculation takes about 15 to $30 \mathrm{~s}$ per mode. The new model is then injected in the predictive part of the LQG controller, to compensate to the most recent vibrations. In the first iteration of our controller, the identification is performed every minute or so, which is enough for slow varying vibrations. But for the faster features described in Secs. 3.1 and 3.2, it is not quite enough. An updated version of the code will allow us to identify the vibration more often, about every $15 \mathrm{~s}$.

\subsection{Loop Correction}

The tip/tilt correction with the PyWFS is decorrelated from the correction all the other modes, which is done using Graphical Processing Units (GPUs) and a simple integrator controller. ${ }^{13}$ The LQG controller for the tip/tilt modes is just running on a Central Processing Unit (CPU). The LQG control loop was tested during onsky observations over several nights. Figure 11 presents two examples of correction by the LQG. The left figure shows the correction of vibrations in a calm case where telescope vibrations are low, and the right figure shows the correction of the strong $5 \mathrm{~Hz}$ vibration around the transit time. In both cases, we can see that vibrations are fully corrected in the closed-loop PSDs. In the first example, telescope vibrations around 5 and $10 \mathrm{~Hz}$, as well as instrument vibrations around $60 \mathrm{~Hz}$ (probably from the image rotator), are mitigated in the closed-loop PSD. The "bump" around $200 \mathrm{~Hz}$ is either an overshoot of AO188's tip/tilt control, or an overshoot of higher modes in the PyWFS control, leaking in the tip and tilt modes. In the second example, The vibration seen during transit at $5 \mathrm{~Hz}$ is fully corrected, leaving a very flat PSD in closed-loop.

Figure 12 presents an evolution of the PSD of the pseudo-open-loop (left) and closed-loop (right) tilt measurement. When we compare the two figures, it looks like the telescope vibrations present in the left between 5 and $10 \mathrm{~Hz}$ are canceled most of the time in the right. In closed-loop, we can see that the model identification was not always finding the same vibrations, creating this discrete effect in the correction. However, the pseudo-open-loop PSD evolution does not show the same effect, which means that the reconstruction of the pseudo-open-loop data is working properly. Finally, this result shows that, thanks to the model identification loop, we are able to track vibrations with slowly varying frequencies.
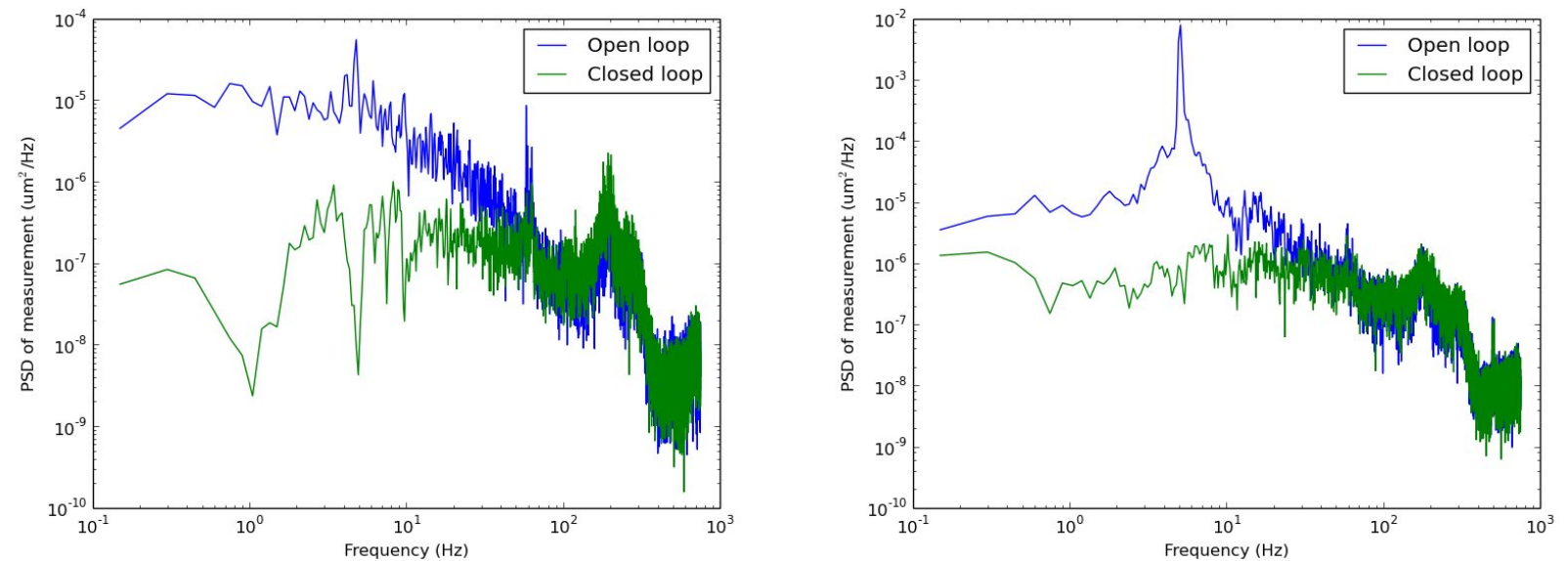

Figure 11. PSD in pseudo-open-loop and closed-loop of the tilt mode, when using the LQG controller, for an average case (left) and a strong transit vibration (right). 

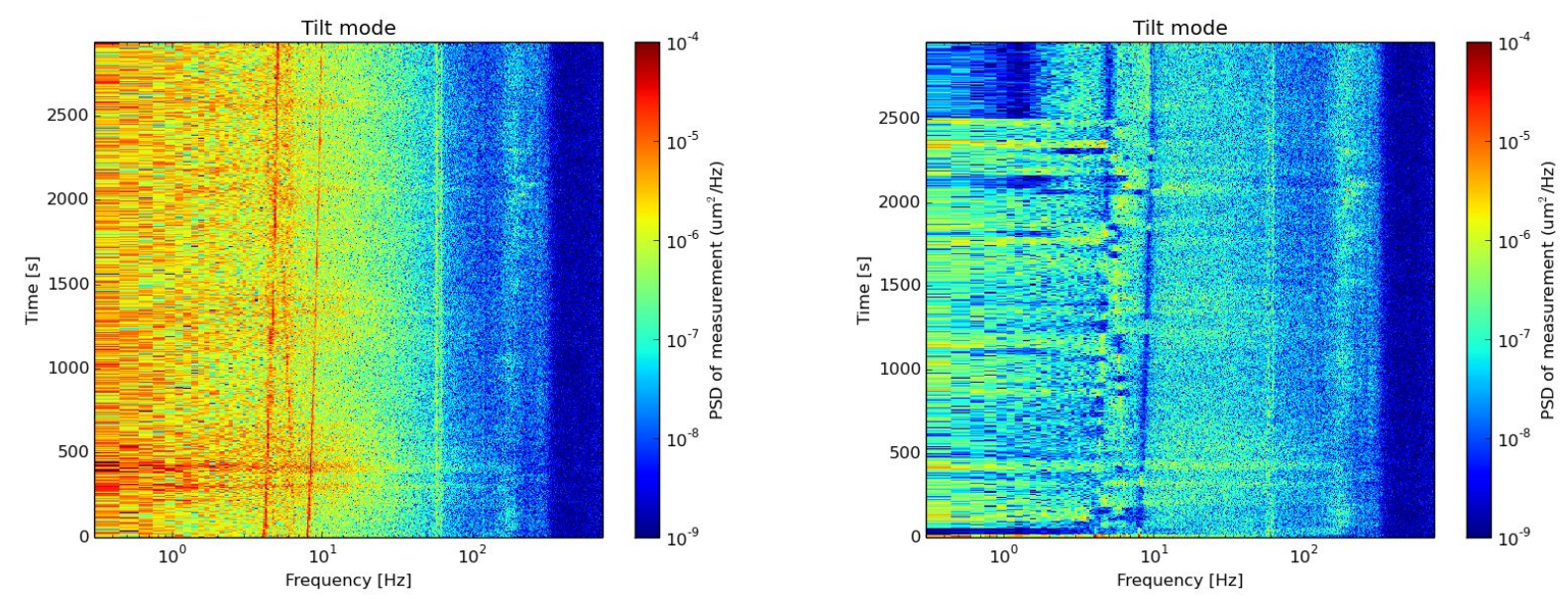

Figure 12. PSD evolution of the tilt mode in pseudo open-loop (left) and closed-loop (right), during average vibrating conditions.

\subsection{Correction of the Vibrations During Transit}

As described in Sec. 3, the strongest feature is the vibration between 4 and $6 \mathrm{~Hz}$ around the time of transit of the target. In this section, we will focus on analyzing its correction by the LQG. By looking at the evolution of the PSD in Fig. 5, we could see that the frequency of the vibration was evolving rapidly at first, then plateaued around $5 \mathrm{~Hz}$ for some time, then decreased rapidly again. The amplitude of the vibration follows the same variations: sharp increase, plateau, then sharp decrease. But if we look at the time series in open-loop, like the one presented in Fig. 13 (left), we can see that the pointing is also affected by sudden high-amplitude jumps, so high that they are usually outside the linearity range of the PyWFS. But in this case, since we are reconstructing open-loop data supposing that the sensor is linear, we can have a good idea of the real amplitude of these jumps. In that figure, we can see that in closed-loop, the tilt residual is fairly constant over that period, except for the few residual jumps. This is confirmed by Fig. 13 (right), which presents a moving standard deviation of the measurements in tip and tilt, in pseudo-open-loop and closed-loop. Here, despite the amplitude and frequency variations of the vibration in the open-loop, the standard deviation of the closed-loop residual is almost constant. Unfortunately, the transients are not fully corrected by the LQG, since they last only a few seconds. We still have to understand what is causing them, and if we can mitigate them at the source.
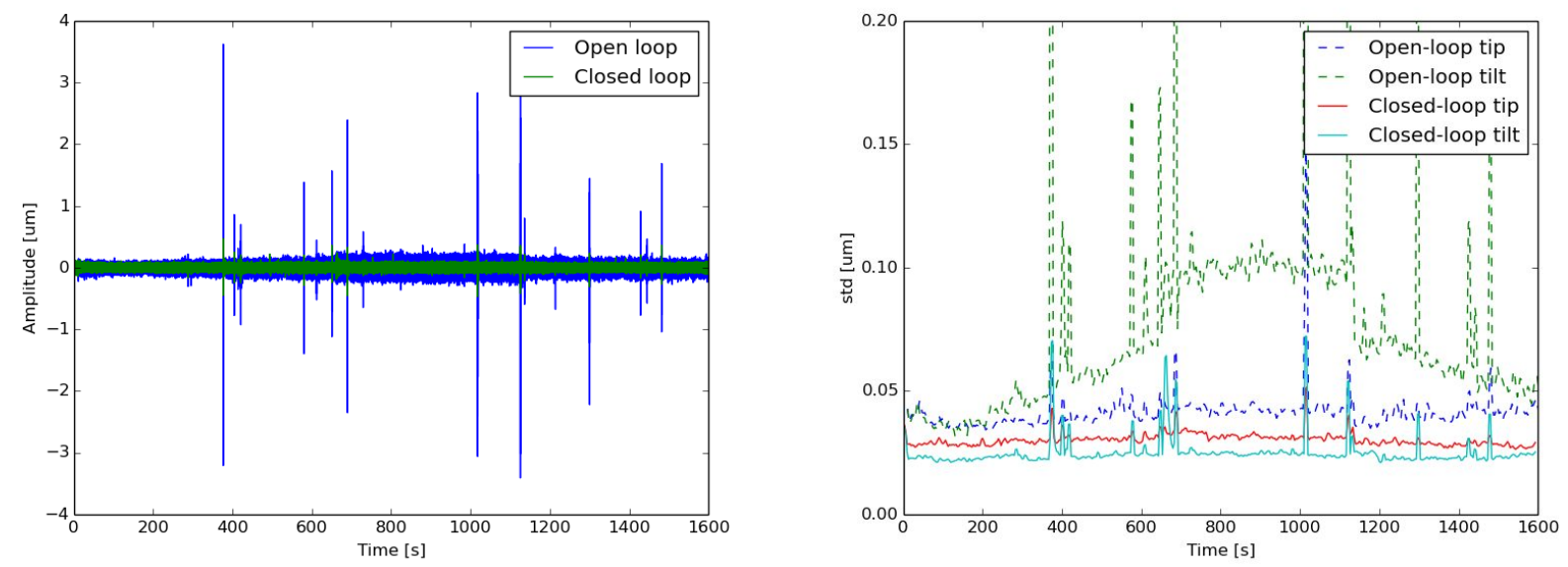

Figure 13. PSD evolution of the pseudo open- and closed-loop tilt (left), and moving standard deviation of the same data (right), around the transit period. 

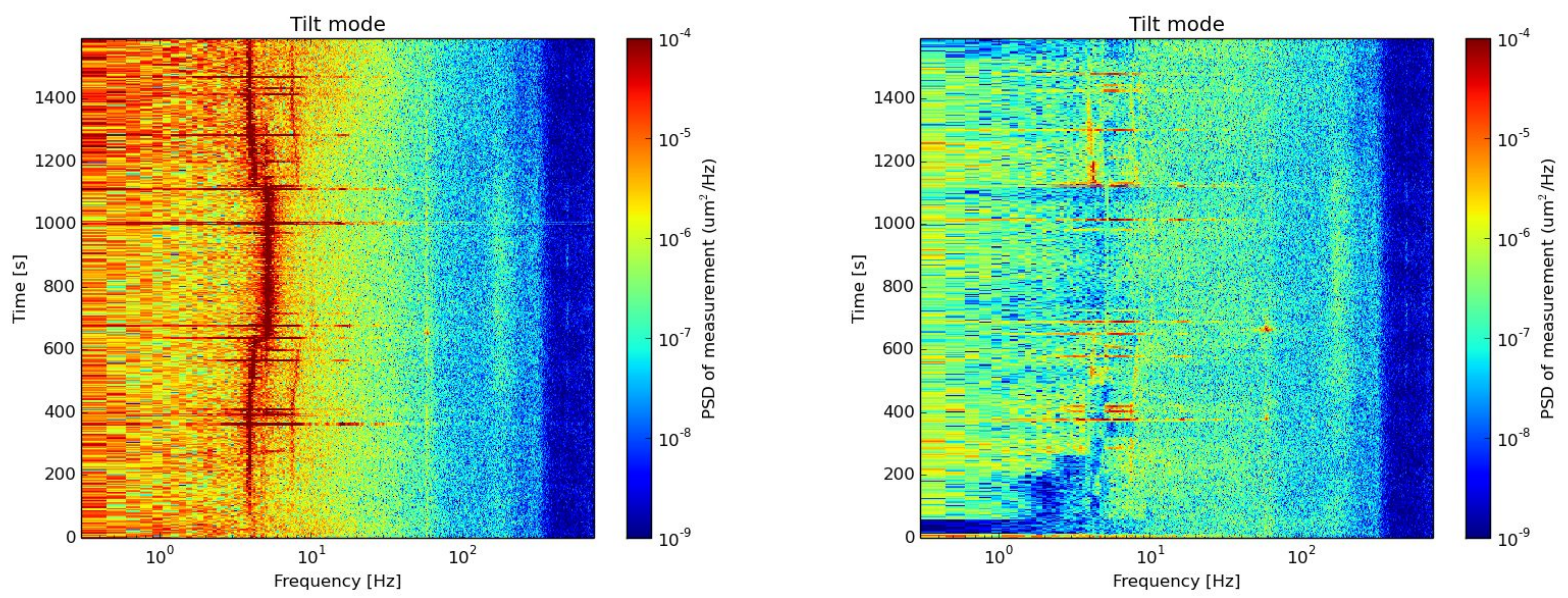

Figure 14. PSD evolution of the tilt in pseudo open-loop (left) and closed-loop (right), around the transit period.

Figure 14 presents the evolution of the PSD for the same data, in pseudo open-loop (left), and closed-loop (right). This figure shows again a pretty good correction of the transit feature, but we can notice that the model identification had trouble keeping up with the frequency variation of the vibration, around $t=600 \mathrm{~s}$ when the plateau starts, and mostly at $t=1100 \mathrm{~s}$, after the plateau stops. It takes a couple of minutes before the new frequency is identified and the model is sent to the LQG controller. Accelerating the model identification loop will make the LQG more responsive to these sharp change in vibration frequencies.

\section{CONCLUSION}

SCExAO, like any other high-contrast imager, SCExAO is very sensitive to vibrations. They reduce the performance of the wavefront control, they degrade science acquisitions, and weaken detection limits of planets, disks and other circumstellar structures. In this paper, using accelerometers and fast sensors, we managed to find the source of most of our vibrations. We reduced vibrations caused by the image rotator of AO188. We also traced down the source of low-frequency vibrations to the telescope drive, and in particular one specific tracking loop. Because of the way the Subaru Telescope is maintained, it will be hard to take care of these vibrations at the source. But such an analysis will help to avoid the same trouble on the similar drive that will be used by the Thirty Meter Telescope. We also demonstrated that an LQG controller coupled with a real-time model identification loop can measure and mitigate vibrations with changing frequencies, stably and over long periods of time. Some improvements will be made on different aspects of the controller - cadence of the identification, optimization of the parameters-- but also a second LQG loop will be implemented with the LLOWFS, to track any non-common residual vibration.

\section{ACKNOWLEDGMENTS}

The authors acknowledge support from the JSPS (Grant-in-Aid for Research \#23340051 \& \#26220704), as well as the help of the Telescope Division team and the AO188 team for their help.

\section{REFERENCES}

[1] Kulcsár, C., Sivo, G., Raynaud, H.-F., Neichel, B., Rigaut, F., Christou, J., Guesalaga, A., Correia, C., Véran, J.-P., Gendron, E., Vidal, F., Rousset, G., Morris, T., Esposito, S., Quiros-Pacheco, F., Agapito, G., Fedrigo, E., Pettazzi, L., Clare, R., Muradore, R., Guyon, O., Martinache, F., Meimon, S., and Conan, J.-M., "Vibrations in AO control: a short analysis of on-sky data around the world," in [Adaptive Optics Systems III], Proc. Soc. Photo-Opt. Instrum. Eng. 8447, 84471C (July 2012). 
[2] Hess, M., Nance, C. E., Vause, J. W., Hrynevych, M., Swain, M. R., and Colavita, M., "Strategy for identifying and mitigating facility vibrations to improve optical performance at the W.M. Keck Observatory," in [Large Ground-based Telescopes], Oschmann, J. M. and Stepp, L. M., eds., Proc. Soc. Photo-Opt. Instrum. Eng. 4837, 342-351 (Feb. 2003).

[3] Brix, M., Naranjo, V., Beckmann, U., Bertram, R., Bertram, T., Brynnel, J., Egner, S., Gaessler, W., Herbst, T. M., Kuerster, M., Rohloff, R. R., Rost, S., and Schmidt, J., "Vibration measurements at the Large Binocular Telescope (LBT)," in [Ground-based and Airborne Telescopes II], Proc. Soc. Photo-Opt. Instrum. Eng. 7012, 70122J (July 2008).

[4] Hartung, M., Hayward, T., Saddlemyer, L., Poyneer, L., Cardwell, A., Cavedoni, C., Cho, M., Chilcote, J. K., Collins, P., Dillon, D., Galvez, R., Gausachs, G., Goodsell, S., Guesalaga, A., Hibon, P., Larkin, J., Macintosh, B., Palmer, D., Sadakuni, N., Savransky, D., Serio, A., Rantakyrö, F., and Wallace, K., "On-sky vibration environment for the Gemini Planet Imager and mitigation effort," in [Adaptive Optics Systems IV], Proc. Soc. Photo-Opt. Instrum. Eng. 9148, 91480N (Aug. 2014).

[5] Jovanovic, N., Guyon, O., Martinache, F., Clergeon, C., Singh, G., Kudo, T., Newman, K., Kuhn, J., Serabyn, E., Norris, B., Tuthill, P., Stewart, P., Huby, E., Perrin, G., Lacour, S., Vievard, S., Murakami, N., Fumika, O., Minowa, Y., Hayano, Y., White, J., Lai, O., Marchis, F., Duchene, G., Kotani, T., and Woillez, J., "Development and recent results from the Subaru coronagraphic extreme adaptive optics system," in [Ground-based and Airborne Instrumentation for Astronomy V], Proc. Soc. Photo-Opt. Instrum. Eng. 9147, 91471Q (July 2014).

[6] Jovanovic, N., Martinache, F., Guyon, O., Clergeon, C., Singh, G., Kudo, T., Garrel, V., Newman, K., Doughty, D., Lozi, J., Males, J., Minowa, Y., Hayano, Y., Takato, N., Morino, J., Kuhn, J., Serabyn, E., Norris, B., Tuthill, P., Schworer, G., Stewart, P., Close, L., Huby, E., Perrin, G., Lacour, S., Gauchet, L., Vievard, S., Murakami, N., Oshiyama, F., Baba, N., Matsuo, T., Nishikawa, J., Tamura, M., Lai, O., Marchis, F., Duchene, G., Kotani, T., and Woillez, J., "The Subaru Coronagraphic Extreme Adaptive Optics System: Enabling High-Contrast Imaging on Solar-System Scales," Pub. Astron. Soc. Pacific 127, 890-910 (Oct. 2015).

[7] Kanzawa, T., Tomono, D., Usuda, T., Takato, N., Negishi, S., Sugahara, S., and Itoh, N., "Improvement of the pointing accuracy of the Subaru Telescope by suppressing vibrations," in [Society of Photo-Optical Instrumentation Engineers (SPIE) Conference Series], Proc. Soc. Photo-Opt. Instrum. Eng. 6267, 62673J (June 2006).

[8] Norris, B. R. M., Tuthill, P. G., Ireland, M. J., Lacour, S., Zijlstra, A. A., Lykou, F., Evans, T. M., Stewart, P., Bedding, T. R., Guyon, O., and Martinache, F., "Probing dusty circumstellar environments with polarimetric aperture-masking interferometry," in [Optical and Infrared Interferometry III], Proc. Soc. Photo-Opt. Instrum. Eng. 8445, 844503 (July 2012).

[9] Huby, E., Perrin, G., Marchis, F., Lacour, S., Kotani, T., Duchêne, G., Choquet, E., Gates, E. L., Woillez, J. M., Lai, O., Fédou, P., Collin, C., Chapron, F., Arslanyan, V., and Burns, K. J., "FIRST, a fibered aperture masking instrument. I. First on-sky test results," Astron. Astrophys. 541, A55 (May 2012).

[10] Atkinson, D., Hall, D., Baranec, C., Baker, I., Jacobson, S., and Riddle, R., "Observatory deployment and characterization of SAPHIRA HgCdTe APD arrays," in [High Energy, Optical, and Infrared Detectors for Astronomy VI], Proc. Soc. Photo-Opt. Instrum. Eng. 9154, 915419 (July 2014).

[11] Singh, G., Martinache, F., Baudoz, P., Guyon, O., Matsuo, T., Jovanovic, N., and Clergeon, C., "Lyotbased Low Order Wavefront Sensor for Phase-mask Coronagraphs: Principle, Simulations and Laboratory Experiments," Pub. Astron. Soc. Pacific 126, 586-594 (June 2014).

[12] Singh, G., Lozi, J., Guyon, O., Baudoz, P., Jovanovic, N., Martinache, F., Kudo, T., Serabyn, E., and Kuhn, J., "On-Sky Demonstration of Low-Order Wavefront Sensing and Control with Focal Plane Phase Mask Coronagraphs," Pub. Astron. Soc. Pacific 127, 857-869 (Oct. 2015).

[13] Lozi, J., Guyon, O., Jovanovic, N., Males, J., Martinache, F., Singh, G., Pathak, P., and Goebel, G., "High precision wavefront control using multiple wavefront sensors: the SCExAO experience," in [Adaptive Optics Systems V], Proc. Soc. Photo-Opt. Instrum. Eng. 9909, 990919 (June 2016).

[14] Petit, C., Conan, J.-M., Kulcsár, C., Raynaud, H.-F., and Fusco, T., "First laboratory validation of vibration filtering with LQG control law for Adaptive Optics," Opt. Express 16, 87 (2008). 
[15] Sivo, G., Kulcsár, C., Conan, J.-M., Raynaud, H.-F., Gendron, É., Basden, A., Vidal, F., Morris, T., Meimon, S., Petit, C., Gratadour, D., Martin, O., Hubert, Z., Sevin, A., Perret, D., Chemla, F., Rousset, G., Dipper, N., Talbot, G., Younger, E., Myers, R., Henry, D., Todd, S., Atkinson, D., Dickson, C., and Longmore, A., "First on-sky SCAO validation of full LQG control with vibration mitigation on the CANARY pathfinder," Opt. Express 22, 23565 (Sept. 2014).

[16] Meimon, S., Petit, C., Fusco, T., and Kulcsar, C., "Tip-tilt disturbance model identification for Kalmanbased control scheme: application to XAO and ELT systems," J. Opt. Soc. Am. A 27, A122 (Sept. 2010).

[17] Petit, C., Sauvage, J.-F., Fusco, T., Sevin, A., Suarez, M., Costille, A., Vigan, A., Soenke, C., Perret, D., Rochat, S., Barrufolo, A., Salasnich, B., Beuzit, J.-L., Dohlen, K., Mouillet, D., Puget, P., Wildi, F., Kasper, M., Conan, J.-M., Kulcsár, C., and Raynaud, H.-F., "SPHERE eXtreme AO control scheme: final performance assessment and on sky validation of the first auto-tuned LQG based operational system," in [Adaptive Optics Systems IV], Proc. Soc. Photo-Opt. Instrum. Eng. 9148, 91480 O (Aug. 2014). 